\title{
Geomagnetic constraints on stratification at the top of Earth's core
}

\author{
David Gubbins \\ School of Earth and Environment, University of Leeds, Leeds LS2 9JT, UK \\ (Received October 5, 2007; Revised March 28, 2007; Accepted May 16, 2007; Online published July 20, 2007)
}

\begin{abstract}
The geodynamo requires that at least part of the Earth's liquid core be convecting so vigorously as to mix it thoroughly to uniform composition and adiabatic temperature. It is possible, however, that part of liquid core is stably stratified, either thermally because of a low temperature gradient or compositionally because light material has separated out. The top of the outer core is the most likely site for stability because the adiabat is steepest there and because light material will rise to the surface. Here I show that part of the observed secular variation, that associated with flux expulsion in the southern hemisphere, can only be caused by fluid upwelling in the electromagnetic boundary layer at the top of the core or by very strong poloidal field gradients at the top of the core. Any stratified layer is limited to roughly the uppermost $100 \mathrm{~km}$ if flux expulsion is the mechanism; if the layer is any thicker the region in the southern hemisphere where reverse flux patches are growing must also be the site of very strong field gradients caused by very strong near-surface shear flows.
\end{abstract}

Key words: Geomagnetism, stratification, core convection, secular variation.

\section{Introduction}

Core convection is driven by vertical gradients in temperature and composition. At depths where the Earth's core convects it is well mixed: the composition is uniform to a very high degree and the temperature is very close to the adiabat. Convection may penetrate into regions where the core is stratified, mixing the fluid everywhere. If the stratification is strong, however, vertical motion is completely inhibited and vertical mixing may not occur, even though horizontal motions may be present. Some seismic observations have suggested stratification at the top of the core (Lay and Young, 1990; Helffrich and Kaneshima, 2004; Eaton and Kendall, 2006). These studies make direct inference of density gradients and therefore detect regions of strong stratification where there is no vertical mixing. In this paper I use the term stratification to mean strong stratification where there is no vertical motion, at least on the temporal and spatial scales likely to influence the magnetic field.

Thermal convection occurs when the conduction gradient exceeds the adiabat. The adiabatic gradient is given by

$$
T_{\mathrm{ad}}^{\prime}=-\frac{g T \gamma}{\phi}
$$

where $g$ is acceleration of gravity, $T$ is temperature, $\gamma$ is the Grüneisen parameter, and $\phi$ is the seismic parameter. $g / \phi$ increases strongly with radius (Dziewonski and Anderson, 1981) and causes the adiabat to steepen towards the core surface; $\gamma$ and $T$ are less well known but will certainly not decrease with radius by enough to cause the adiabat to shallow. The conduction gradient depends on the distribution of heat sources: if basal heating dominates, as would occur if

Copyright (c) The Society of Geomagnetism and Earth, Planetary and Space Sciences (SGEPSS); The Seismological Society of Japan; The Volcanological Society of Japan; The Geodetic Society of Japan; The Japanese Society for Planetary Sciences; TERRAPUB. the main source of heat were latent heat of freezing of the core at the inner core boundary (ICB), the conduction gradient is steepest at the bottom; if internal heating dominates, as would occur if radiogenic heat sources were strong in the liquid core, it could steepen towards the top. In either event the most likely site for a thermally stratified layer is at the top of the core, where the adiabatic gradient is steep and the conduction gradient shallow.

Chemical convection is driven by light material released on freezing at the ICB, which may either mix with the bulk liquid of the outer core or, depending on the solubility properties with pressure, rise to form a layer at the top of the liquid core (Fearn and Loper, 1981; Helffrich and Kaneshima, 2004). The top of the core is therefore also the most likely place to find chemical stratification.

The density gradient in a stratified region is given by

$$
\frac{d \rho}{d r}=-\frac{\rho g}{\phi}+\alpha \rho \tau+\alpha_{c} \rho \tau_{c},
$$

where $\tau$ is the subadiabatic temperature gradient

$$
\tau=\left.\frac{d T}{d r}\right|_{\mathrm{ad}}-\frac{d T}{d r},
$$

$\tau_{c}$ is the compositional gradient

$$
\tau_{c}=\frac{d c}{d r},
$$

$\alpha$ is the coefficient of thermal expansion, and $\alpha_{c}$ is the coefficient of compositional volume expansion

$$
\alpha_{c}=\frac{1}{\rho}\left(\frac{\partial \rho}{\partial c}\right)_{P, T} .
$$

In a convecting region $\tau$ and $\tau_{c}$ are effectively zero and Eq. (2) becomes the Adams-Williamson equation. Since $g$ 
depends only on $\rho$ it is possible to integrate this equation for density using the measured seismic parameter, and many density models have been obtained in this way. A stable layer can, in principle, be detected by its effect on seismic body waves or free oscillations. Normal mode eigenfrequencies are affected by the bouyancy force and are therefore sensitive to the stratification, but only weakly so. The extreme case of an isothermal core gives only an $8 \%$ departure from the neutrally stable density gradient, while normal modes can only detect a $12 \%$ departure in density with a spatial resolution of $400 \mathrm{~km}$ (Masters and Gubbins, 2003). Composition has a greater effect on the density than it does on temperature and a layer enriched in light elements could be detected if it were sufficiently light and extensive, but so far none has been observed using normal modes. A stratified layer can be inferred indirectly from anomalously low seismic velocity because bulk modulus is relatively insensitive to composition in a liquid; three recent seismological studies find evidence of a thin, low-density layer just below the core-mantle boundary (CMB) (Lay and Young, 1990; Helffrich and Kaneshima, 2004; Eaton and Kendall, 2006).

It also is possible, in principle, to detect stratification at the top of the core from geomagnetic secular variation (SV) using the frozen flux theory of Roberts and Scott (1965) and Backus (1968). Setting the electrical diffusivity of the core to zero leaves an evolution equation for the radial component of magnetic field in terms of the fluid flow at the $\mathrm{CMB}$. This equation predicts zero $\mathrm{SV}$ at extrema of the field, and Whaler (1980) found that the contour of zero SV passed remarkably close to all the extrema. A second consequence of frozen flux theory is that all integrals of the $\mathrm{SV}$ over patches on the CMB bounded by contours of radial field should be zero in the absence of radial motion. This provides a better test because estimates of integrals over patches have realistic error estimates whereas the location of extrema do not (Backus, 1988); Whaler (1980) again found no evidence for upwelling, although at that time there were no reliable estimates for the errors on the integrals. Later Whaler (1986) concluded there was evidence for fluid upwelling at the top of the core by simply fitting a purely toroidal flow to an SV model and finding the fit to be unacceptable. Other workers have also argued for upwelling (Madden and Mouël, 1982; Gire et al., 1986; Voorhies, 1986) and yet more have obtained good fits to SV without upwelling (Bloxham, 1986, 1989; Lloyd and Gubbins, 1990). Later work has focussed on flows with some dynamical consistency, notably flows with tangential geostrophy (Mouël, 1984; Mouël et al., 1985; Gire and Le Mouël, 1990). These assume no magnetic forces close to the CMB and allow upwelling. Bloxham (1990) found it possible to fit SV data with purely zonal toriodal motions and horizontally polarised MAC waves. Recently, Pais et al. (2004) have found improved fits to SV by relaxing the geostrophic constraint and allowing for some magnetic forces.

All the above geomagnetic tests of stratification rely on the frozen flux hypothesis. In this paper I argue that magnetic diffusion vitiates these studies while at the same time placing a useful constraint on stratification in the uppermost $100 \mathrm{~km}$ or so of the core.
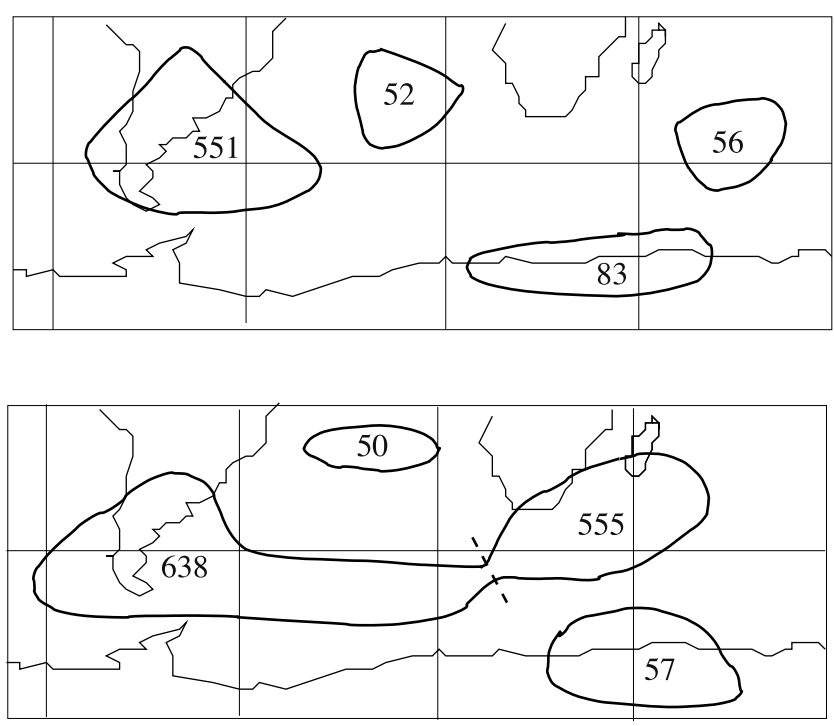

Fig. 1. Reverse flux patches and total integrated flux (in MWb) on the CMB for epoch 1905.5 (upper) and 1969.5 (lower). The main change occurs in the small patch marked 56 in 1905.5, which increases by about $500 \mathrm{MWb}$ in 69 years and merges to form the present "dog's-bone" patch. This behaviour has continued since AD1800 at least, and is a feature of recent satellite models.

\section{Effects of Magnetic Diffusion}

Models of the magnetic field on the core surface show several null lines beneath the South Atlantic and Indian Oceans (Fig. 1). They separate regions where field lines point into the core from regions where they point out of it, the "reverse flux". The flux through these patches has grown significantly over the last few centuries (Bloxham and Gubbins, 1985) and detectably during the last 25 years of satellite observation (Hulot et al., 2002; Wardinski and Holme, 2006). This cannot be explained by frozen-flux theory, which predicts zero change: it requires diffusion.

Reverse-flux patches are thought to form when strong toroidal field is brought near the surface and emerges to form a pair of features similar to sunspots (Allan and Bullard, 1966; Bloxham, 1986). The vertical field $B_{r}$ at the core surface satisfies a component of the induction equation

$$
\begin{aligned}
&\left(\frac{\partial}{\partial t}-\right.\left.\eta \nabla^{2}\right)\left(r B_{r}\right)= \\
& r\left(\boldsymbol{B} \cdot \nabla_{\mathrm{h}} v_{r}-\boldsymbol{v} \cdot \nabla_{\mathrm{h}} B_{r}+B_{r} v_{r}^{\prime}-v_{r} B_{r}^{\prime}\right)
\end{aligned}
$$

where $\boldsymbol{v}, \boldsymbol{B}$ denote velocity and magnetic field respectively; $r, \theta, \phi$ are the spherical coordinates; $\eta$ is the electrical diffusion; $\nabla_{\mathrm{h}}$ is the horizontal gradient; and prime denotes differentiation with respect to $r$. The operator on the left hand side can be inverted in the form of a Green's function that decays exponentially with depth into the core; the typical form is the same as for heat diffusion: $\exp \left[-\eta\left(t-t_{1}\right) /\left|\boldsymbol{r}-\boldsymbol{r}_{1}\right|^{2}\right]$ (Gubbins, 1996). The Green's function is concentrated around the observation point $\boldsymbol{r}$ and time $t$. The exponential decays in a distance comparable with the electromagnetic skin depth corresponding to the observation time, $\delta_{w}=\sqrt{\eta\left(t-t_{1}\right)}$.

The relevant term on the right hand side of (6) for toroidal flux expulsion is $r \boldsymbol{B}_{T} \cdot \nabla_{\mathrm{h}} v_{r}$, where $\boldsymbol{B}_{T}$ is the toroidal part 
of the field given in terms of the toroidal scalar $\mathcal{T}$

$$
\boldsymbol{B}_{T}=\nabla \times(\mathcal{T} \boldsymbol{r})
$$

Retaining only this term on the right hand side of Eq. (6) and integrating over a patch $S_{0}$ of the core surface bounded by a null-flux curve gives the change in flux over time $t$

$$
\begin{aligned}
\Delta \int_{S_{0}} B_{r} d S & =\frac{\eta}{c} \int_{S_{0}} \int_{0}^{t}\left(t-t_{1}\right) \frac{1}{\sin \theta} \\
& \times\left(\frac{\partial \mathcal{T}^{\prime}}{\partial \phi} \frac{\partial v_{r}^{\prime}}{\partial \theta}-\frac{\partial \mathcal{T}^{\prime}}{\partial \theta} \frac{\partial v_{r}^{\prime}}{\partial \phi}\right) d t_{1} d S
\end{aligned}
$$

where $c$ is the core radius (Gubbins, 1996). This equation can be used to estimate the vertical gradient of the toroidal field within the electromagnetic skin depth at the top of the core; everything else is known from observation or can be estimated from the frozen flux determination of the fluid velocity.

A rough numerical estimate of the quantities using $\eta=$ $1.6 \mathrm{~m}^{2} \mathrm{~s}^{-1}, c=3.485 \times 10^{6} \mathrm{~m}, l=10^{6} \mathrm{~m}$ (the horizontal length scale), $A=3 \times 10^{12} \mathrm{~m}^{2}$ (the area of the patch), $v_{r}^{\prime}=0.02$ /year (from core motion inversion, e.g. Jackson and Bloxham (1991)), $t=69$ years, and left hand side $500 \mathrm{MWb}$ (Fig. 1), gives $\mathcal{T}^{\prime} \approx 20 \mathrm{nT} \mathrm{m}^{-1}$. This applies within the electromagnetic skin depth corresponding to 69 years, which is $\delta_{w}=60 \mathrm{~km}$. At this depth the toroidal field reaches about $1 \mathrm{mT}$, or twice the poloidal field at the core surface. This is a reasonable value for the toroidal field, and shows toroidal flux expulsion to be a viable explanation for the increase in flux through this reversed flux patch.

If there is no radial motion in the vicinity of the CMB then $v_{r}^{\prime}$ is zero as well as $v_{r}$ and toroidal flux expulsion does not operate. The only term remaining on the right hand side of (6) is the second, $-r v \cdot \nabla_{\mathrm{h}} B_{r}$. Integrating (6) over a patch bounded by a null-flux curve removes the term involving the fluid velocity to leave

$$
\frac{d}{d t} \int_{S_{0}} r B_{r} d S=\eta \int_{S_{0}} \nabla^{2}\left(r B_{r}\right) d S .
$$

Once again we estimate the term on the left hand side by the flux difference divided by the time interval. For the right hand side we need an estimate of $\nabla^{2}\left(r B_{r}\right)$ near the top of the core. This contains first and second derivatives of $B_{r}$, all of which can be estimated from downward continued surface data except for $B_{r}^{\prime \prime}$, which must be discontinuous across the CMB because $\nabla^{2}\left(r B_{r}\right)=0$ in the insulating mantle but not in the conducting core. It will be shown later that observed horizontal derivatives are not large enough to account for the change in flux through the patch, so we require $B_{r}^{\prime \prime}$ to be large in the sense $B_{r}^{\prime \prime} \gg B_{r}^{\prime} / c$ and estimate $\nabla^{2}\left(r B_{r}\right)$ by $c B_{r}^{\prime \prime}$. A rough estimate of (9) is then

$$
\Delta \int_{S_{o}} B_{r} d S \approx \eta t A B_{r}^{\prime \prime}
$$

Taking the same numerical values as before gives $B_{r}^{\prime \prime} \approx$ $5 \times 10^{-14} \mathrm{~T} \mathrm{~m}^{-2}$. Comparing this value with estimates of $B^{\prime}{ }_{r}$ obtained by downward continuing surface data gives a length scale for the vertical rate of change in $B_{r}$. Taking $\boldsymbol{B}_{\mathrm{h}} \approx 5 \times 10^{-4} \mathrm{~T}$ gives $B^{\prime}{ }_{r}=\boldsymbol{B}_{\mathrm{h}} / l \approx 5 \times 10^{-10} \mathrm{~T}$ $\mathrm{m}^{-1}$. The vertical length scale is then $B^{\prime}{ }_{r} / B_{r}^{\prime \prime} \approx 10 \mathrm{~km}$. This is much shorter than the horizontal length scale $l$, justifying our neglect of other derivatives. It is also an order of magnitude smaller than the electromagnetic skin depth, showing the necessary gradients to be very steep. Surface changes caused by diffusion will only be significant if there is considerable shearing of the poloidal field just below the core surface.

\section{Discussion}

Changes in the reverse-flux patches have been large throughout the $20^{\text {th }}$ century, so large as to be comparable with changes in the other flux patches used to infer upwelling. This suggests the error in estimates of upwelling using the frozen-flux approximation is large, so large that we are not justified in attributing these aspects of SV to upwelling rather than diffusion; nor are we justified in ruling out toroidal motions on the grounds of a poor fit to observations when the misfit might equally well be caused by diffusion. Other dangers of using the frozen-flux approximation have been highlighted in a rather different context by Gubbins and Kelly (1996), Love (1999), and, perhaps most convincingly, by Alfvén himself (Alfvén, 1943).

There are only 2 viable mechanisms to explain the changes in reverse flux patches if the top of the core is stratified: expulsion of toroidal field by radial motion beneath a rather thin stratified layer or very steep gradients of poloidal field near the surface. Both are possible, although the shearing must be severe in order to produce such strong gradients in poloidal field and toroidal flux expulsion seems the more likely.

Toroidal field and vertical motion are both zero at the $\mathrm{CMB}$, and expulsion relies on generating poloidal flux by the inductive effect of vertical motion on toroidal field in shallow regions and allowing the poloidal field to diffuse out. Induction in the shallowest regions influences surface changes the most, the effect decaying exponentially with depth $x$ into the core as $\exp -x^{2} / \delta_{w}^{2}$. Strong shears can exist in the stratified layer and these can produce strong toroidal fields; if vertical motion below the stratified layer acts on these toroidal fields they can produce the observed changes in flux at the surface. The critical depth, $\delta_{w}$, depends on the electrical conductivity, $\sigma$, and the strength of the toroidal field within the core. The result is relatively insensitive to the choice of $\sigma$; increasing or decreasing it by a factor of 2 only changes the critical depth of the layer by $40 \%$. The secular variation observations therefore constrain any stable layer to the top $100 \mathrm{~km}$ or so of the core at most. Another uncertainty arises from diffusion effects on small scale magnetic fields that are not resolved by surface data. This is a serious concern when dealing with point estimates of the field on the CMB, but for this study we have used integrals of $B_{r}$ over patches that should be large enough to be well resolved by surface data and average out effects of small scale fields. Furthermore, the diffusive term is linear in $B_{r}$ and its derivatives, so the diffusive effects of small scale fields should also average out.

If toroidal flux expulsion is not possible because of stratification, very strong gradients in $B_{r}$ are required involving exponential growth of $B_{r}$ into the core, reaching $0.2 \mathrm{~T}$ 
(2000 Gauss) at the bottom of the electromagnetic skin depth. This seems unlikely, but the estimated field strength is very sensitive to the estimated length scale. Such a large field gradient requires strong shear flow.

This study shows that expulsion of toroidal flux from the core requires vertical flow within $100 \mathrm{~km}$ of the core surface. The only other mechanism capable of explaining the recent changes in flux through the core surface in the southern hemisphere involves steep gradients in radial field and concomitant high shear immediately beneath the core surface. The results are based on kinematic arguments and observations; dynamical studies are required for further exploration of the two possible mechanisms for flux changes.

Acknowledgments. This work was supported by the DESMond Consortium funded by NERC grant NER/O/S/2001/006668.

\section{References}

Alfvén, H., On the existence of electromagnetic-hydromagnetic waves, Arkiv. Mat. Astron. Fys, 29B(2)7, 1943.

Allan, D. W. and E. C. Bullard, The secular variation of the Earth's magnetic field, Proc. Camb. Phil. Soc., 62, 783-809, 1966.

Backus, G. E., Kinematics of the secular variation, Philos. Trans. R. Soc. London Ser. A, 263, 239-266, 1968.

Backus, G. E., Bayesian inference in geomagnetism, Geophys. J., 92, 125142, 1988.

Bloxham, J., The expulsion of flux from the Earth's core, Geophys. J. R. Astron. Soc., 87, 669-678, 1986.

Bloxham, J., Simple models of fluid flow at the core surface derived from geomagnetic field models, Geophys. J. R. Astr. Soc., 99, 173-182, 1989.,

Bloxham, J., On the consequences of strong stable stratification at the top of Earth's outer core, Geophys. Res. Lett., 17, 2081-2084, 1990.

Bloxham, J. and D. Gubbins, The secular variation of the Earth's magnetic field, Nature, 317, 777-781, 1985.

Dziewonski, A. M. and D. L. Anderson, Preliminary Reference Earth Model, Phys. Earth Planet. Int., 25, 297-356, 1981.

Eaton, D. W. and J.-M. Kendall, Improving seismic resolution of outermost core structure by multichannel analysis and deconvolution of broadband SmKS phases, Phys. Earth Planet. Int., 155, 104-109, 2006.

Fearn, D. R. and D. E. Loper, Compositional convection and stratification of Earth's core, Nature, 289, 393-394, 1981.

Gire, C. and J.-L. Le Mouël, Tangentially geostrophic flow at the coremantle boundary compatible with the observed secular variation: The large-scale component of flow, Phys. Earth Planet. Int., 59, 259-287, 1990.
Gire, C., J.-L. L. Mouël, and T. Madden, Motions of the core surface derived from SV data, Geophys. J. R. Astron. Soc., 84, 1-29, 1986.

Gubbins, D., A formalism for the inversion of geomagnetic data for core motions with diffusion, Phys. Earth Planet. Int., 98, 193-206, 1996.

Gubbins, D. and P. Kelly, A difficulty with using the frozen flux hypothesis to find steady core motions, Geophys. Res. Lett., 23, 1825-1828, 1996.

Helffrich, G. and S. Kaneshima, Seismological consraints on core composition from Fe-O-S liquid immiscibility, Science, 306, 2239-2242, 2004.

Hulot, G., C. Eymin, B. Langlais, M. Mandea, and N. Olsen, Small-scale structure of the geodynamo inferred from Oersted and Magsat satellite data, Nature, 416, 620-623, 2002.

Jackson, A. and J. Bloxham, Mapping the fluid flow and shear near the core surface using the radial and horizontal components of the magnetic field, Geophys. J. Int., 105, 199-212, 1991.

Lay, T. and C. J. Young, The stably-stratified outermost core revisited, Geophys. Res. Lett., 17, 2001-2004, 1990.

Lloyd, D. B. and D. Gubbins, Toroidal fluid motions at the top of the Earth's core, Geophys. J. R. Astr. Soc., 100, 455-467, 1990.

Love, J. J., A critique of frozen-flux inverse modelling of a nearly steady geodynamo, Geophys. J. Int., 138, 353-365, 1999.

Madden, T. and J.-L. L. Mouël, The recent secular variation and the motions at the core surface, Philos. Trans. R. Soc. London Ser. A, 306, 271-280, 1982.

Masters, T. G. and D. Gubbins, On the resolution of density within the Earth, Phys. Earth Planet. Int., 140, 159-167, 2003.

Mouël, J.-L. L., Outer core geostrophic flow and secular variation of Earth's geomagnetic field, Nature, 311, 734-735, 1984.

Mouël, J.-L. L., C. Gire, and T. Madden, Motions of the core surface in the geostrophic approximation, Phys. Earth Planet. Int., 39, 270-287, 1985.

Pais, M. A., O. Oliveira, and F. Nogueira, Nonuniqueness of inverted coremantle boundary flows and deviations from tangential geostrophy, $J$. Geophys. Res., 109, doi:10.1029/2004/22004JB003012, 2004.

Roberts, P. H. and S. Scott, On the analysis of the secular variation. A hydromagnetic constraint: I. theory, J. Geomagn. Geoelectr., 17, 137$151,1965$.

Voorhies, C. V., Steady flows at the top of Earth's core derived from geomagnetic field models, J. Geophys. Res., 15, 12,444-12,466, 1986.

Wardinski, I. and R. Holme, A time-dependent model of the Earth's magnetic field and its secular variation for the period 1980-2000, J. Geophys. Res., 111, doi:10.1029/2006JB004401, 2006.

Whaler, K. A., Does the whole of the Earth's core convect?, Nature, 287, 528-530, 1980.

Whaler, K. A., Geomagnetic evidence for fluid upwelling at the coremantle boundary, Geophys. J. R. Astr. Soc., 86, 563-588, 1986.

D. Gubbins (e-mail: ear6dg@earth.leeds.ac.uk) 